\title{
ON SYMMETRIC GENERALIZED 3-DERIVATIONS AND COMMUTATIVITY IN PRIME NEAR-RINGS
}

\author{
Kyoo-Hong Park And Yong-SoO Jung*
}

\begin{abstract}
In this note, we introduce a symmetric generalized 3derivation in near-rings and investigate some conditions for a nearring to be a commutative ring.
\end{abstract}

\section{Introduction and preliminaries}

A non-empty set $R$ with two binary operations + (addition) and . (multiplication) is called a near-ring if it satisfies the following axioms:

i) $(R,+)$ is a group (not necessarily abelian),

ii) $(R, \cdot)$ is a semigroup,

iii) $x \cdot(y+z)=x \cdot y+x \cdot z$ for all $x, y, z \in R$.

Precisely speaking, it is a left near-ring because it satisfies the left distributive law. We will use the word near-ring to mean left near-ring and denote $x y$ instead of $x \cdot y$.

For a near-ring $R$, the set $R_{0}=\{x \in R: 0 x=0\}$ is called the zero-symmetric part of $R$. A near-ring $R$ is said to be zero-symmetric if $R=R_{0}$. Throughout this note, $R$ will be a zero-symmetric near-ring and $R$ is called prime if $x R y=\{0\}$ implies $x=0$ or $y=0$. Recall that $R$ is called $n$-torsion-free, where $n$ is a positive integer, if $n x=0$ implies $x=0$ for all $x \in R$. The symbol $C$ will represent the multiplicative center of $R$, that is, $C=\{x \in R: x y=y x$ for all $y \in R\}$. For $x \in R$, the symbol $C(x)$ will denote the centralizer of $x$ in $R$. As usual, for $x, y \in R$, $[x, y]$ will denote the commutator $x y-y x$, while $\langle x, y\rangle$ will indicate the additive-group commutator $x+y-x-y$. As for terminologies concerning near-rings used here without special mention, we refer to G. Pilz [10].

An additive map $d: R \rightarrow R$ is called a derivation if the Leibniz rule $d(x y)=d(x) y+x d(y)$ holds for all $x, y \in R$. By a bi-derivation we mean

Received March 9, 2009. Accepted June 2, 2009.

2000 Mathematics Subject Classification: 16W20, 16Y30,16Y99.

Keywords and phrases : prime near-ring, commutative ring, symmetric 3derivation, symmetric generalized 3-derivation.

${ }^{*}$ Corresponding author. 
a bi-additive map $D: R \times R \rightarrow R$ (i.e., $D$ is additive in both arguments) which satisfies the relations

$$
D(x y, z)=D(x, z) y+x D(y, z)
$$

and

$$
D(x, y z)=D(x, y) z+y D(x, z)
$$

for all $x, y, z \in R$. Let $D$ be symmetric, that is, $D(x, y)=D(y, x)$ for all $x, y \in R$. The map $\tau: R \rightarrow R$ defined by $\tau(x)=D(x, x)$ for all $x, y \in R$ is called the trace of $D$.

Derivations and bi-derivations in rings and near-rings have been studied by many mathematicians in several ways $[1,2,3,4,5,6,7,8,11$, 12, 15, 16]. Furthermore, M. Uçkun and M.A. Öztürk [14] investigated symmetric bi- $\Gamma$-derivations and commutativity in $\Gamma$-near-rings.

A 3-additive map $\Delta: R \times R \times R \rightarrow R$ is called a 3-derivation if the relations

$$
\begin{aligned}
\Delta\left(x_{1} x_{2}, y, z\right) & =\Delta\left(x_{1}, y, z\right) x_{2}+x_{1} \Delta\left(x_{2}, y, z\right), \\
\Delta\left(x, y_{1} y_{2}, z\right) & =\Delta\left(x, y_{1}, z\right) y_{2}+y_{1} \Delta\left(x, y_{2}, z\right)
\end{aligned}
$$

and

$$
\Delta\left(x, y, z_{1} z_{2}\right)=\Delta\left(x, y, z_{1}\right) z_{2}+z_{1} \Delta\left(x, y, z_{2}\right)
$$

are fulfilled for all $x, y, z, x_{i}, y_{i}, z_{i} \in R, i=1,2$.

Let a map $F: R \times R \times R \rightarrow R$ be symmetric, namely, the equation $F\left(x_{1}, x_{2}, x_{3}\right)=F\left(x_{\pi(1)}, x_{\pi(2)}, x_{\pi(3)}\right)$ holds for all $x_{1}, x_{2}, x_{3} \in R$ and for every permutation $\{\pi(1), \pi(2), \pi(3)\}$. A map $f: R \rightarrow R$ defined by $f(x)=F(x, x, x)$ for all $x \in R$, is the trace of $F$. It is obvious that, in the case when $F: R \times R \times R \rightarrow R$ is a symmetric map which is also 3 -additive (i.e., additive in each argument), the trace $f$ of $F$ satisfies the relation

$f(x+y)=f(x)+2 F(x, x, y)+F(x, y, y)+F(x, x, y)+2 F(x, y, y)+f(y)$ for all $x, y \in R$.

Since we have

$$
F(0, y, z)=F(0+0, y, z)=F(0, y, z)+F(0, y, z)
$$

for all $y, z \in R$, we obtain $F(0, y, z)=0$ for all $y, z \in R$. Hence we get

$$
0=F(0, y, z)=F(x-x, y, z)=F(x, y, z)+F(-x, y, z)
$$

and so we see that $F(-x, y, z)=-F(x, y, z)$ for all $x, y, z \in R$. This tells us that $f$ is an odd function. If $\Delta$ is symmetric, then the above three relations are equivalent to each other. 
An additive map $h: R \rightarrow R$ is called a generalized derivation if there exists a derivation $d: R \rightarrow R$ such that $h(x y)=d(x) y+x h(y)$ for all $x, y \in R$. M. Brešar [4] defined this concept in rings. This notion is found in [13] and other properties of generalized derivations in rings and near-rings were given by B. Hvala [9], H.E. Bell [2] and Ö. Gölbaşi [8], respectively.

A symmetric bi-additive map $H: R \times R \rightarrow R$ is said to be a symmetric generalized bi-left derivation (resp.symmetric generalized bi-right derivation) associated with $D$ if there exists a symmetric bi-derivation $D: R \times R \rightarrow R$ such that $H(x y, z)=D(x, z) y+x H(y, z)$ (resp. $H(x y, z)=H(x, z) y+x D(y, z))$ for all $x, y, z \in R$. $H$ is called a symmetric generalized bi-derivation associated with $D$ if it is both a symmetric generalized bi-left derivation and a symmetric generalized bi-right derivation associated with $D$ (see [14]).

Here we introduce the following map:

By a symmetric generalized 3-left derivation (resp. symmetric generalized 3-right derivation) associated with $\Delta$, we mean a symmetric 3-additive map $\mathcal{G}: R \times R \times R \rightarrow R$ such that there exists a symmetric 3-derivation $\Delta: R \times R \times R \rightarrow R$ satisfying $\mathcal{G}(x y, z, w)=\Delta(x, z, w) y+$ $x \mathcal{G}(y, z, w)$ (resp. $\mathcal{G}(x y, z, w)=\mathcal{G}(x, z, w) y+x \Delta(y, z, w))$ for all $w, x, y, z \in$ $R$. The map $\mathcal{G}$ is called a symmetric generalized 3 -derivation associated with $\Delta$ if it is both a symmetric generalized 3-left derivation and a symmetric generalized 3-right derivation associated with $\Delta$. We here will denote the generalized 3-(left or right) derivation $\mathcal{G}$ associated with the symmetric 3 -derivation $\Delta$ by the tuple $(\mathcal{G}, \Delta)$. Note that the symmetric generalized 3-derivation $(\Delta, \Delta)$ is the symmetric 3-derivation $\Delta$.

As a simple example, let $N$ be a commutative near-ring and let

$$
R=\left\{\left(\begin{array}{cc}
a & b \\
0 & 0
\end{array}\right) \mid a, b \in N\right\} .
$$

Then it is easy to see that $R$ is a near-ring under matrix addition and matrix multiplication and that the map $\Delta: R \times R \times R \rightarrow R$ defined by

$$
\left(\left(\begin{array}{cc}
a_{1} & b_{1} \\
0 & 0
\end{array}\right),\left(\begin{array}{cc}
a_{2} & b_{2} \\
0 & 0
\end{array}\right),\left(\begin{array}{cc}
a_{3} & b_{3} \\
0 & 0
\end{array}\right)\right) \mapsto\left(\begin{array}{cc}
0 & a_{1} a_{2} a_{3} \\
0 & 0
\end{array}\right)
$$

is a symmetric 3 -derivation.

Furthermore, we define a map $\mathcal{G}: R \times R \times R \rightarrow R$ by

$$
\left(\left(\begin{array}{cc}
a_{1} & b_{1} \\
0 & 0
\end{array}\right),\left(\begin{array}{cc}
a_{2} & b_{2} \\
0 & 0
\end{array}\right),\left(\begin{array}{cc}
a_{3} & b_{3} \\
0 & 0
\end{array}\right)\right) \mapsto\left(\begin{array}{cc}
0 & a_{1} a_{2} a_{3}+b_{1} b_{2} b_{3} \\
0 & 0
\end{array}\right) .
$$


Then $(\mathcal{G}, \Delta)$ is a symmetric generalized 3-left derivation.

In this note, we investigate the conditions for a near-ring with symmetric generalized 3 -derivations to be a commutative ring.

\section{Lemmata}

We need the following lemmas to obtain our main results.

Lemma 2.1 ([3, Lemma 3]). Let $R$ be a prime near-ring. If $C \backslash\{0\}$ contains an element $z$ for which $z+z \in C$, then $(R,+)$ is abelian.

Lemma 2.2. Let $R$ be a 2 and 3-torsion free near-ring. Suppose that there exists a symmetric 3-additive map $F: R \times R \times R \rightarrow R$ such that $f(x)=0$ for all $x \in R$, where $f$ is the trace of $F$. Then we have $F=0$.

Proof. For any $x, y \in R$,

$f(x+y)=f(x)+2 F(x, x, y)+F(x, y, y)+F(x, x, y)+2 F(x, y, y)+f(y)$ and so, by the hypothesis, we get

$$
2 F(x, x, y)+F(x, y, y)+F(x, x, y)+2 F(x, y, y)=0
$$

for all $x, y \in R$. Putting $-x$ instead of $x$ in (2.1), we obtain

$$
2 F(x, x, y)-F(x, y, y)+F(x, x, y)-2 F(x, y, y)=0
$$

for all $x, y \in R$.

On the other hand, for any $x, y \in R$,

$f(y+x)=f(y)+2 F(y, y, x)+F(y, x, x)+F(y, y, x)+2 F(y, x, x)+f(x)$ and thus, by the hypothesis, we have

$$
2 F(x, y, y)+F(x, x, y)+F(x, y, y)+2 F(x, x, y)=0
$$

for all $x, y \in R$ since $F$ is symmetric. Comparing (2.1) with (2.2), we get

$$
2 F(x, y, y)+F(x, x, y)+F(x, y, y)=F(x, x, y)-3 F(x, y, y)
$$

which implies that

$$
\begin{aligned}
& 2 F(x, y, y)+F(x, x, y)+F(x, y, y)+2 F(x, x, y) \\
= & F(x, x, y)-3 F(x, y, y)+2 F(x, x, y)
\end{aligned}
$$

for all $x, y \in R$. Hence it follows from (2.3) that

$$
F(x, x, y)-3 F(x, y, y)+2 F(x, x, y)=0
$$

for all $x, y \in R$. The substitution $x=-x$ in (2.4) leads to

$$
F(x, x, y)+3 F(x, y, y)+2 F(x, x, y)=0
$$


for all $x, y \in R$. Combining (2.4) and (2.5), we obtain

$$
F(x, y, y)=0
$$

for all $x, y \in R$ since $R$ is 6 -torsion free. The replacement $y=y+z$ to linearize (2.6) yields

$$
F(x, y, z)=0
$$

for all $x, y, z \in R$, i.e., $F=0$ which completes the proof.

Lemma 2.3. Let $R$ be a 2 and 3-torsion free prime near-ring and let $x \in R$. Suppose that there exists a nonzero symmetric generalized 3-derivation $(\mathcal{G}, \Delta): R \times R \times R \rightarrow R$ such that $x g(y)=0$ for all $y \in R$, where $g$ is the trace of $\mathcal{G}$. Then we have $x=0$.

Proof. Since we have

$g(y+z)=g(y)+2 \mathcal{G}(y, y, z)+\mathcal{G}(y, z, z)+\mathcal{G}(y, y, z)+2 \mathcal{G}(y, z, z)+g(z)$ for all $y, z \in R$, the hypothesis gives

$$
2 x \mathcal{G}(y, y, z)+x \mathcal{G}(y, z, z)+x \mathcal{G}(y, y, z)+2 x \mathcal{G}(y, z, z)=0
$$

for all $y, z \in R$. Setting $y=-y$ in (2.7), it follows that

$$
2 x \mathcal{G}(y, y, z)-x \mathcal{G}(y, z, z)+x \mathcal{G}(y, y, z)-2 x \mathcal{G}(y, z, z)=0
$$

for all $y, z \in R$.

On the other hand, for any $y, z \in R$,

$g(z+y)=g(z)+2 \mathcal{G}(z, z, y)+\mathcal{G}(z, y, y)+\mathcal{G}(z, z, y)+2 \mathcal{G}(z, y, y)+g(y)$ and so, by the hypothesis, we have

$$
2 x \mathcal{G}(y, z, z)+x \mathcal{G}(y, y, z)+x \mathcal{G}(y, z, z)+2 x \mathcal{G}(y, y, z)=0
$$

for all $y, z \in R$ since $\mathcal{G}$ is symmetric. Comparing (2.8) with (2.9), we get

$$
2 x \mathcal{G}(y, z, z)+x \mathcal{G}(y, y, z)+x \mathcal{G}(y, z, z)=x \mathcal{G}(y, y, z)-3 x \mathcal{G}(y, z, z)
$$

which means that

$$
\begin{aligned}
& 2 x \mathcal{G}(y, z, z)+x \mathcal{G}(y, y, z)+x \mathcal{G}(y, z, z)+2 x \mathcal{G}(y, y, z) \\
= & x \mathcal{G}(y, y, z)-3 x \mathcal{G}(y, z, z)+2 x \mathcal{G}(y, y, z)
\end{aligned}
$$

for all $y, z \in R$. Now, from (2.9), we obtain

$$
x \mathcal{G}(y, y, z)-3 x \mathcal{G}(y, z, z)+2 x \mathcal{G}(y, y, z)=0
$$

for all $y, z \in R$. Taking $y=-y$ in (2.10) leads to

$$
x \mathcal{G}(y, y, z)+3 x \mathcal{G}(y, z, z)+2 x \mathcal{G}(y, y, z)=0
$$


for all $y, z \in R$. Combining (2.10) and (2.11), we obtain

$$
x \mathcal{G}(y, z, z)=0
$$

for all $y \in R$ since $R$ is 6 -torsion free. Replacing $z=z+w$ to linearize (2.12) and using the conditions show that

$$
x \mathcal{G}(w, y, z)=0
$$

for all $w, y, z \in R$. Assume first that $\Delta=0$. Since $(\mathcal{G}, \Delta)$ is a nonzero symmetric generalized 3-left derivation, substituting $w v$ for $w$ in (2.13) implies that

$$
x w \mathcal{G}(v, y, z)=0
$$

for all $v, w, y, z \in R$. By the primeness of $R$, we get $x=0$. On the other hand, assume that $\Delta \neq 0$. Since $(\mathcal{G}, \Delta)$ is also a symmetric generalized 3 -right derivation, again substituting $w v$ for $w$ in (2.13) implies that

$$
x w \Delta(v, y, z)=0
$$

for all $v, w, y, z \in R$. From the primeness of $R$, we have $x=0$ which gives the conclusion.

Lemma 2.4. Let $R$ be a near-ring and let $(\mathcal{G}, \Delta): R \times R \times R \rightarrow R$ be a symmetric generalized 3-derivation. Then for all $v, w, x, y, z \in R$, we have, respectively,

(i) $[\mathcal{G}(x, z, w) y+x \Delta(y, z, w)] v=\mathcal{G}(x, z, w) y v+x \Delta(y, z, w) v$;

(ii) $[\Delta(x, z, w) y+x \mathcal{G}(y, z, w)] v=\Delta(x, z, w) y v+x \mathcal{G}(y, z, w) v$.

Proof. (i) Since we have

$$
\mathcal{G}(x y, z, w)=\mathcal{G}(x, z, w) y+x \Delta(y, z, w)
$$

for all $w, x, y, z \in R$, the associative law gives

$$
\begin{aligned}
\mathcal{G}((x y) v, z, w) & =\mathcal{G}(x y, z, w) v+x y \Delta(v, z, w) \\
& =[\mathcal{G}(x, z, w) y+x \Delta(y, z, w)] v+x y \Delta(v, z, w)
\end{aligned}
$$

for all $v, w, x, y, z \in R$ and

$$
\begin{aligned}
\mathcal{G}(x(y v), z, w) & =\mathcal{G}(x, z, w) y v+x \Delta(y v, z, w) \\
& =\mathcal{G}(x, z, w) y v+x[\Delta(y, z, w) v+y \Delta(v, z, w)] \\
& =\mathcal{G}(x, z, w) y v+x \Delta(y, z, w) v+x y \Delta(v, z, w)
\end{aligned}
$$

for all $v, w, x, y, z \in R$. Comparing (2.14) and (2.15), we see that

$$
[\mathcal{G}(x, z, w) y+x \Delta(y, z, w)] v=\mathcal{G}(x, z, w) y v+x \Delta(y, z, w) v
$$

for all $v, w, x, y, z \in R$.

(ii) It is proved by the similar way to (i). 


\section{Main results}

Now we are ready to prove our main results.

Theorem 3.1. Let $R$ be a 2 and 3-torsion free prime near-ring. Suppose that there exists a nonzero symmetric generalized 3-derivation $(\mathcal{G}, \Delta): R \times R \times R \rightarrow R$ such that

$$
\mathcal{G}(x, y, z) \in C
$$

for all $x, y, z \in R$. Then $R$ is a commutative ring.

Proof. Assume that $\mathcal{G}(x, y, z) \in C$ for all $x, y, z \in R$. Since $(\mathcal{G}, \Delta)$ is nonzero, there exist $x_{0}, y_{0}, z_{0} \in R$ such that $\mathcal{G}\left(x_{0}, y_{0}, z_{0}\right) \in C \backslash\{0\}$ and

$$
\mathcal{G}\left(x_{0}, y_{0}, z_{0}\right)+\mathcal{G}\left(x_{0}, y_{0}, z_{0}\right)=\mathcal{G}\left(x_{0}, y_{0}, z_{0}+z_{0}\right) \in C .
$$

Hence $(R,+)$ is abelian by Lemma 2.1 .

Let $\Delta=0$. Then $\mathcal{G}(x y, z, w)=\mathcal{G}(x, z, w) y \in C$ holds for all $w, x, y \in$ $R$ and hence we have $\mathcal{G}(x, z, w) y v=v \mathcal{G}(x, z, w) y$ for all $v, w, x, y, z \in R$ which implies that $[y, v] g(x)=0$ for all $v, x, y \in R$. Reminding of Lemma 2.3 , we get $[y, v]=0$ for all $v, y \in R$, i.e., $R$ is a commutative ring.

Let $\Delta \neq 0$. Since the hypothesis implies that

$$
w \mathcal{G}(x, y, z)=\mathcal{G}(x, y, z) w
$$

for all $w, x, y, z \in R$, we replace $x$ by $x v$ in (3.1) to get

$$
w[\mathcal{G}(x, y, z) v+x \Delta(v, y, z)]=[\mathcal{G}(x, y, z) v+x \Delta(v, y, z)] w
$$

and thus, from Lemma 2.4(i) and the hypothesis, it follows that

$$
\mathcal{G}(x, y, z) w v+w x \Delta(v, y, z)=\mathcal{G}(x, y, z) v w+x \Delta(v, y, z) w
$$

which means that

$$
\mathcal{G}(x, y, z)[w, v]=[x \Delta(v, y, z), w]
$$

for all $v, w, x, y, z \in R$. Since $(\mathcal{G}, \Delta)$ is a symmetric generalized 3-left derivation and $g(x) \in C$, setting $g(x) u$ in place of $x$ in (3.2) and using Lemma 2.4(ii) yield

$$
\Delta(g(x), y, z) u[w, v]+g(x) \mathcal{G}(u, y, z)[w, v]=g(x)[u \Delta(v, y, z), w]
$$

for all $u, w, x, y, z \in R$. So the relation (3.2) gives

$$
\Delta(g(x), y, z) u[w, v]=0
$$

for all $u, w, x, y, z \in R$. 
Suppose that $R$ is not commutative. Since $R$ is prime, we get, from (3.3),

$$
\Delta(g(x), y, z)=0
$$

for all $x, y, z \in R$. Let us $x=x+w$ in (3.4). Then we have

$$
\begin{aligned}
0 & =\Delta(g(x+w), y, z) \\
& =\Delta(g(x)+g(w)+3 \mathcal{G}(x, x, w)+3 \mathcal{G}(w, w, x), y, z) \\
& =3 \Delta(\mathcal{G}(x, x, w), y, z)+3 \Delta(\mathcal{G}(x, w, w), y, z),
\end{aligned}
$$

that is,

$$
\Delta(\mathcal{G}(x, x, w), y, z)+\Delta(\mathcal{G}(x, w, w), y, z)=0
$$

for all $w, x, y, z \in R$. Letting $x=-x$ in (3.5) and then comparing the result with (3.5), we see that

$$
\Delta(\mathcal{G}(x, x, w), y, z)=0
$$

for all $w, x, y, z \in R$. We replace $x$ by $x+v$ in (3.6) to obtain

$$
\Delta(\mathcal{G}(x, v, w), y, z)=0
$$

for all $v, w, x, y, z \in R$. Substituting $x u$ for $x$ in (3.7), we get

$$
\begin{aligned}
0= & \Delta(\mathcal{G}((x u, v, w), y, z) \\
= & \Delta(\mathcal{G}(x, v, w) u+x \Delta(u, v, w), y, z)) \\
= & \Delta(\mathcal{G}(x, v, w) u, y, z)+\Delta(x \Delta(u, v, w), y, z) \\
= & \Delta(\mathcal{G}(x, v, w), y, z) u+\mathcal{G}(x, v, w) \Delta(u, y, z) \\
& +\Delta(x, y, z) \Delta(u, v, w)+x \Delta(\Delta(u, v, w), y, z)
\end{aligned}
$$

and so the equality (3.7) gives

$$
\mathcal{G}(x, v, w) \Delta(u, y, z)+\Delta(x, y, z) \Delta(u, v, w)+x \Delta(\Delta(u, v, w), y, z)=0
$$

for all $u, v, w, x, y, z \in R$. Let us write $g(w)$ in place of $w$ in (3.8). Then it follows from (3.4) that

$$
\mathcal{G}(g(w), x, v) \Delta(u, y, z)=0
$$

for all $u, v, w, x, y, z \in R$. Putting $t u$ in substitute for $u$ in (3.9) yields

$$
\mathcal{G}(g(w), x, v) t \Delta(u, y, z)=0
$$

for all $t, u, v, w, x, y, z \in R$ which implies that

$$
\mathcal{G}(g(w), x, v)=0
$$


for all $v, x, w \in R$ by the primeness of $R$ and $\Delta \neq 0$. Taking $y+w$ instead of $w$ in (3.10), we get

$$
\begin{aligned}
0 & =\mathcal{G}(g(y+w), x, v) \\
& =\mathcal{G}(g(y)+g(w)+3 \mathcal{G}(y, y, w)+3 \mathcal{G}(y, w, w), x, v) \\
& =3 \mathcal{G}(\mathcal{G}(y, y, w), x, v)+3 \mathcal{G}(\mathcal{G}(y, w, w), x, v),
\end{aligned}
$$

that is,

$$
\mathcal{G}(\mathcal{G}(y, y, w), x, v)+\mathcal{G}(\mathcal{G}(y, w, w), x, v)=0
$$

for all $v, w, x, y \in R$. Setting $y=-y$ in (3.11) and then combining the result with (3.11), we see that

$$
\mathcal{G}(\mathcal{G}(y, y, w), x, v)=0
$$

for all $v, w, x, y \in R$. We replace $y$ by $y+z$ in (3.12) to obtain

$$
\mathcal{G}(\mathcal{G}(y, z, w), x, v)=0
$$

for all $v, w, x, y, z \in R$. Substituting $y u$ for $y$ in (3.13) gives the relation

$$
\begin{aligned}
0= & \mathcal{G}(\mathcal{G}((y u, z, w), x, v) \\
= & \mathcal{G}(\Delta(y, z, w) u+y \mathcal{G}(u, z, w), x, v)) \\
= & \mathcal{G}(\Delta(y, z, w) u, x, v)+\mathcal{G}(y \mathcal{G}(u, z, w), x, v) \\
= & \mathcal{G}(\Delta(y, z, w), x, v) u+\Delta(y, z, w) \Delta(u, x, v) \\
& +\Delta(y, x, v) \mathcal{G}(u, z, w)+y \mathcal{G}(\mathcal{G}(u, z, w), x, v)
\end{aligned}
$$

from which the equality (3.13) leads to the relation

$$
\mathcal{G}(\Delta(y, z, w), x, v) u+\Delta(y, z, w) \Delta(u, x, v)+\Delta(y, x, v) \mathcal{G}(u, z, w)=0
$$

for all $u, v, w, x, y, z \in R$. Letting $u=g(u)$ in (3.14), we have, by (3.4) and (3.10),

$$
\mathcal{G}(\Delta(y, z, w), x, v) g(u)=0
$$

for all $u, v, w, x, y, z \in R$. Now, from Lemma 2.3 and $\mathcal{G} \neq 0$, the equality (3.15) implies that

$$
\mathcal{G}(\Delta(y, z, w), x, v)=0
$$

for all $v, w, x, y, z \in R$ and putting $y=z=w$ in (3.16) yields

$$
\mathcal{G}(\delta(y), x, v)=0
$$

for all $v, x, y \in R$. Hence, by (3.16), the equality (3.14) reduces to the relation

$$
\Delta(y, z, w) \Delta(u, x, v)+\Delta(y, x, v) \mathcal{G}(u, z, w)=0
$$


for all $u, v, w, x, y, z \in R$. Replacing $u$ by $\delta(u)$ in (3.18), the equality (3.17) tells us that

$$
\Delta(y, z, w) \Delta(\delta(u), x, v)=0
$$

for all $u, v, w, x, y, z \in R$ and so taking $z t$ instead of $z$ in (3.19) gives

$$
\Delta(y, z, w) t \Delta(\delta(u), x, v)=0
$$

for all $t, u, v, w, x, y, z \in R$. The primeness of $R$ now leads to

$$
\Delta(\delta(u), x, v)=0
$$

for all $u, v, x \in R$ in view of $\Delta \neq 0$. By (3.20), we see that

$$
\Delta(\delta(u+x), u+x, u+x)+\Delta(\delta(-u+x),-u+x,-u+x)=0
$$

for all $u, x \in R$ from which we use the relation (3.20) to get

(3.21) $\Delta(\Delta(u, u, x), u, u)+\Delta(\Delta(u, u, x), x, x)+2 \Delta(\Delta(u, x, x), u, x)=0$

for all $u, x \in R$. Writing $u+x$ for $x$ in (3.21) and utilizing (3.20), we obtain

$$
0=6 \Delta(\Delta(u, u, x), u, u)+6 \Delta(\Delta(u, u, x), u, x)+\Delta(\Delta(u, u, x), x, x)
$$

$$
+2 \Delta(\Delta(u, x, x), u, u)+2 \Delta(\Delta(u, x, x), u, x)
$$

for all $u, x \in R$ and so substituting $-u$ for $u$ in (3.22) and combining the result with (3.23) yield

$$
\Delta(\Delta(u, x, x), u, u)+3 \Delta(\Delta(u, u, x), u, x)=0
$$

for all $u, x \in R$. Taking $u=u+x$ in (3.23) and using (3.20), we have

$$
0=\Delta(\Delta(u, x, x), u, u)+3 \Delta(\Delta(u, u, x), u, x)+3 \Delta(\Delta(u, u, x), x, x)
$$

$$
+3 \Delta(\Delta(u, x, x), u, x)+3 \Delta(\Delta(u, x, x), x, x)
$$

for all $u, x \in R$. Let us replace $x$ by $-x$ in (3.24) and compare the result with (3.24). Then we obtain

$$
\Delta(\Delta(u, u, x), x, x)+\Delta(\Delta(u, x, x), u, x)=0
$$

for all $u, x \in R$. Putting $u=u+x$ in (3.25) and using (3.20), the relation (3.25) reduces to

(3.26) $\Delta(\Delta(u, u, x), x, x)+3 \Delta(\Delta(u, x, x), x, x)+\Delta(\Delta(u, x, x), u, x)=0$ for all $u, x \in R$. Replacing $x$ by $-x$ in (3.26) and combining the result with (3.26), we have

$$
\Delta(\Delta(u, x, x), x, x)=0
$$


for all $u, x \in R$. We substitute $u x$ for $u$ in (3.27) and then employ (3.20) and (3.27) to arrive at

$$
\Delta(u, x, x) \delta(x)=0
$$

for all $u, x \in R$. Finally, taking $x y$ instead of $u$ in (3.28), it follows that

$$
\delta(x) y \delta(x)=0
$$

for all $x, y \in R$. Since $R$ is prime, we see that $\delta=0$ which implies $\Delta=0$ by Lemma 2.2. This is a contradiction because of $\Delta \neq 0$. Hence $R$ is commutative. This completes the proof.

Theorem 3.2. Let $R$ be a 2 and 3-torsion free prime near-ring. Suppose that there exists a nonzero symmetric generalized 3-derivation $(\mathcal{G}, \Delta): R \times R \times R \rightarrow R$ such that

$$
g(x), g(x)+g(x) \in C(\mathcal{G}(u, v, w))
$$

for all $u, v, w, x, y \in R$, where $g$ is the trace of $\mathcal{G}$. Then $R$ is a commutative ring.

Proof. Assume that

$$
g(x), g(x)+g(x) \in C(\mathcal{G}(u, v, w))
$$

for all $u, v, w, x \in R$. Thus we get, from (3.29),

$$
\begin{aligned}
& \mathcal{G}(u+p, v, w)(g(x)+g(x)) \\
= & (g(x)+g(x)) \mathcal{G}(u+p, v, w) \\
= & (g(x)+g(x))[\mathcal{G}(u, v, w)+\mathcal{G}(p, v, w)] \\
= & (g(x)+g(x)) \mathcal{G}(u, v, w)+(g(x)+g(x)) \mathcal{G}(p, v, w) \\
= & g(x) \mathcal{G}(u, v, w)+g(x) \mathcal{G}(u, v, w)+g(x) \mathcal{G}(p, v, w)+g(x) \mathcal{G}(p, v, w) \\
= & g(x)[\mathcal{G}(u, v, w)+\mathcal{G}(u, v, w)+\mathcal{G}(p, v, w)+\mathcal{G}(p, v, w)]
\end{aligned}
$$

$$
=[\mathcal{G}(u, v, w)+\mathcal{G}(u, v, w)+\mathcal{G}(p, v, w)+\mathcal{G}(p, v, w)] g(x)
$$

for all $p, u, v, w, x \in R$ and

$$
\begin{aligned}
& \mathcal{G}(u+p, v, w)(g(x)+g(x)) \\
= & \mathcal{G}(u+p, v, w) g(x)+\mathcal{G}(u+p, v, w) g(x) \\
= & g(x) \mathcal{G}(u+p, v, w)+g(x) \mathcal{G}(u+p, v, w) \\
= & g(x)[\mathcal{G}(u, v, w)+\mathcal{G}(p, v, w)]+g(x)[\mathcal{G}(u, v, w)+\mathcal{G}(p, v, w)] \\
= & g(x)[\mathcal{G}(u, v, w)+\mathcal{G}(p, v, w)+\mathcal{G}(u, v, w)+\mathcal{G}(p, v, w)] \\
= & {[\mathcal{G}(u, v, w)+\mathcal{G}(p, v, w)+\mathcal{G}(u, v, w)+\mathcal{G}(p, v, w)] g(x) }
\end{aligned}
$$


for all $p, u, v, w, x \in R$. Comparing (3.30) and (3.31), we obtain

$$
\mathcal{G}(\langle u, p\rangle, v, w) g(x)=0
$$

for all $p, u, v, w, x \in R$. From Lemma 2.3, it follows that

$$
\mathcal{G}(\langle u, p\rangle, v, w)=0
$$

for all $p, u, v, w \in R$.

First, let us consider the case $\Delta=0$. Since $(\mathcal{G}, \Delta)$ is a symmetric generalized 3-right derivation, we substitute $u z$ for $u$ and $u p$ for $p$ in (3.32) to get

$$
\begin{aligned}
0 & =\mathcal{G}(u\langle z, p\rangle, v, w) \\
& =\mathcal{G}(u, v, w)\langle z, p\rangle+u \Delta(\langle z, p\rangle, v, w) \\
& =\mathcal{G}(u, v, w)\langle z, p\rangle
\end{aligned}
$$

and so we see that

$$
g(u)\langle z, p\rangle=0
$$

for all $p, u, z \in R$. Letting $z=w z$ and $p=w p$ in (3.33) yields

$$
g(u) w\langle z, p\rangle=0
$$

for all $p, u, z, w \in R$. Since Lemma 2.2 tells us that $\mathcal{G} \neq 0$ implies $g \neq 0$, we conclude, from (3.34) and the primeness of $R$, that $\langle z, p\rangle=0$ is fulfilled for all $p, z \in R$. Therefore $(R,+)$ is abelian.

Since we have $\Delta=0$ and $(\mathcal{G}, \Delta)$ is a symmetric generalized 3-right derivation, we obtain

$$
\mathcal{G}(x y, z, t)=\mathcal{G}(x, z, t) y \in C(\mathcal{G}(u, v, w))
$$

for all $t, u, v, w, x, y, z \in R$. Thus we have

$$
\mathcal{G}(x, z, t) y \mathcal{G}(u, v, w)=\mathcal{G}(u, v, w) \mathcal{G}(x, z, t) y
$$

for all $t, u, v, w, x, y, z \in R$ which implies that

$$
\mathcal{G}(x, z, t)[y, \mathcal{G}(u, v, w)]=0
$$

for all $t, u, v, w, x, y, z \in R$. Taking $x s$ in place of $x$ in this equation, we get

$$
\mathcal{G}(x, z, t) s[y, \mathcal{G}(u, v, w)]=0
$$

for all $s, t, u, v, w, x, y \in R$ since $(\mathcal{G}, \Delta)$ is a symmetric generalized 3-right derivation. Since $R$ is prime and $\mathcal{G} \neq 0$, we obtain $[y, \mathcal{G}(u, v, w)]=0$ for all $u, v, w, y \in R$, i.e, $\mathcal{G}(u, v, w) \in C$ for all $u, v, w \in R$. Invoking Theorem $3.1, R$ is a commutative ring. 
Let $\Delta \neq 0$ and let $\delta$ be the trace of $\Delta$. Since $(\mathcal{G}, \Delta)$ is a symmetric generalized 3-left derivation, replacing $u$ by $u z$ and $p$ by up in (3.32) yields that

$$
\begin{aligned}
0 & =\mathcal{G}(u\langle z, p\rangle, v, w) \\
& =\Delta(u, v, w)\langle z, p\rangle+u \mathcal{G}(\langle z, p\rangle, v, w) \\
& =\Delta(u, v, w)\langle z, p\rangle
\end{aligned}
$$

and so we obtain

$$
\Delta(u, v, w)\langle z, p\rangle=0
$$

for all $p, u, z \in R$. Letting $z=s z$ and $p=s p$ in (3.35) yields

$$
\Delta(u, v, w) s\langle z, p\rangle=0
$$

for all $p, s, u, v, w, z \in R$. Since $\Delta \neq 0$, we conclude, from (3.36) and the primeness of $R$, that $\langle z, p\rangle=0$ is fulfilled for all $p, z \in R$. Hence $(R,+)$ is abelian.

We claim that $R$ is a commutative ring. Indeed, from the hypothesis, we see that

$$
[g(x), \mathcal{G}(u, v, w)]=0
$$

for all $u, v, w, x \in R$. Hence if we let $x=x+y$ in (3.37), then we deduce from (3.37) that

$$
[\mathcal{G}(x, x, y), \mathcal{G}(u, v, w)]+[\mathcal{G}(x, y, y), \mathcal{G}(u, v, w)]=0
$$

for all $u, v, w, x, y \in R$. Setting $y=-y$ into (3.38) and comparing the result with (3.38), we obtain

$$
[\mathcal{G}(x, y, y), \mathcal{G}(u, v, w)]=0
$$

for all $u, v, w, x, y \in R$. Replacing $y$ by $y+z$ in (3.39) and using (3.39), we have

$$
[\mathcal{G}(x, y, z), \mathcal{G}(u, v, w)]=0
$$

since $\mathcal{G}$ is permuting, i.e.,

$$
\mathcal{G}(x, y, z) \mathcal{G}(u, v, w)=\mathcal{G}(u, v, w) \mathcal{G}(x, y, z)
$$

for all $u, v, w, x, y, z \in R$. Taking $u t$ instead of $u$ in (3.40) and applying Lemma 2.4(ii), we obtain

$$
\begin{aligned}
& \Delta(u, v, w) t \mathcal{G}(x, y, z)-\mathcal{G}(x, y, z) \Delta(u, v, w) t \\
& \quad+u \mathcal{G}(t, v, w) \mathcal{G}(x, y, z)-\mathcal{G}(x, y, z) u \mathcal{G}(t, v, w)=0
\end{aligned}
$$


for all $t, u, v, w, x, y, z \in R$. Substituting $g(u)$ for $u$ in (3.41) and then utilizing the hypothesis and (3.40), we get

$$
\Delta(g(u), v, w)[t, \mathcal{G}(x, y, z)]=0
$$

for all $t, u, v, w, x, y, z \in R$. Let us write in (3.42) ws instead of $w$. Then we have

$$
\Delta(g(u), v, w) s[t, \mathcal{G}(x, y, z)]=0
$$

for all $s, t, u, v, w, x, y, z \in R$. Since $R$ is prime, we arrive at either $\Delta(g(u), v, w)=0$ or $[t, \mathcal{G}(x, y, z)]=0$ for all $t, u, v, w, x, y, z \in R$.

Following the same process among $(3.4) \sim(3.28)$ in the proof of Theorem 3.1, the case when $\Delta(g(u), v, w)=0$ holds for all $u, v, w \in R$ leads to the contradiction.

Consequently, we obtain

$$
[t, \mathcal{G}(x, y, z)]=0
$$

for all $t, x, y, z \in R$, i.e, $\mathcal{G}(x, y, z) \in C$ for all $x, y, z \in R$. Therefore, Theorem 3.1 tells us that $R$ is a commutative ring which is complete the proof.

\section{References}

[1] M. Ashraf, A. Ali and S. Ali, $(\sigma, \tau)$-derivations on prime near-rings, Archivum Mathematicum (BRNO), Tomus. 40 (2004), 281-286.

[2] H.E. Bell, On prime near-rings with generalized derivation, Internat. J. Math. \& Math. Sci. 2008 (2008), 1-5.

[3] H.E. Bell and G. Mason, On derivations in near-rings, in Near-Rings and NearFields (Tübingen, 1985), G. Betsch, Ed., vol. 137 of North-Holland Mathematics Studies, pp. 31-35, North-Holland, Amsterdam, The Netherlands, 1987.

[4] M. Brešar, On the distance of the compositions of two derivations to the generalized derivations, Glasgow Math. J. 33 (1991), 89-93.

[5] _ Commuting maps: a survey, Taiwanese J. Math. 8(3) (2004), 361-397.

[6] Y. Çeven and M.A. Öztürk, Some properties of symmetric bi- $(\sigma, \tau)$-derivations in near-rings, Commun. Korean Math. Soc. 22(4) (2007), 487-491.

[7] Ö. Gölbaşi, On prime near-rings with generalized $(\sigma, \tau)$-derivations, Kyungpook Math. J. 45 (2005), 249-254.

[8] _ Notes on prime near-rings with generalized derivation, Southeast Asian Bull. Math. 30(1) (2006), 49-54.

[9] B. Hvala, Generalized derivations in prime rings, Comm. Aigebra., 26(4) (1998), 1147-1166.

[10] G. Pilz, Near-rings, 2nd Ed. North Holland, Amsterdam, 1983.

[11] E.C. Posner, Derivations in prime rings, Proc. Amer. Math. Soc. 8 (1957), 1093-1100.

[12] M.A. Quadri, M.S. Khan and N. Rehman, Generalized derivations and commutativity of prime rings, Indian J. pure appl. Math. 34(9) (2003), 1393-1396. 
[13] P. Ribenboim, Higher order derivations of modules, Portgaliae Math. 39 (1980), 381-397.

[14] M. Uçkun and M.A. Öztürk, On trace of symmetric bi-gamma-derivations in gamma-near-rings, Houston J. Math. 33(2) (2007), 323-339.

[15] J. Vukman, Symmetric bi-derivations on prime and semi-prime rings, Aequationes Math. 38 (1989), 245-254.

[16] _ Two results concerning symmetric bi-derivations on prime rings, Aequationes Math. 40 (1990), 181-189.

\section{Kyoo-Hong Park}

Department of Mathematics Education

Seowon University,

Cheongju, Chungbuk 361-742, Korea

E-mail: parkkh@seowon.ac.kr

Yong-Soo Jung

Department of Mathematics

Sun Moon University,

Asan, Chungnam 336-708, Korea

E-mail: ysjung@sunmoon.ac.kr 